\title{
1 A bacterial symbiont protects honey bees from fungal disease
}

2 Delaney L. Miller ${ }^{1}$, Eric A. Smith ${ }^{1}$, Irene L. G. Newton ${ }^{1 *}$

$3 \quad{ }^{1}$ Department of Biology, Indiana University, Bloomington, Indiana, USA

4 *Author for Correspondence: Irene L. G. Newton, Department of Biology, Indiana University,

5 Bloomington, Indiana, USA, (812) 855-3883, irnewton@indiana.edu 
Fungi are the leading cause of insect disease, contributing to the decline of wild and managed populations ${ }^{1,2}$. For ecologically and economically critical species, such as the

27 European honey bee (Apis mellifera), the presence and prevalence of fungal pathogens can have far reaching consequences, endangering other species and threatening food security $^{3,4,5}$. Our ability to address fungal epidemics and opportunistic infections is currently hampered by the limited number of antifungal therapies ${ }^{6,7}$. Novel antifungal treatments are frequently of bacterial origin and produced by defensive symbionts (bacteria that associate with an animal/plant host and protect against natural enemies ${ }^{89}$. Here we examined the capacity of a honey bee-associated bacterium, Bombella apis, to suppress the growth of fungal pathogens and ultimately protect bee brood (larvae and pupae) from infection. Our results showed that strains of $B$. apis inhibit the growth of two insect fungal pathogens, Beauveria bassiana and Aspergillus flavus, in vitro. This phenotype was recapitulated in vivo; bee brood supplemented with $B$. apis were significantly less likely to be infected by $A$. flavus. Additionally, the presence of $B$. apis reduced sporulation of $A$.

39 flavus in the few bees that were infected. Analyses of biosynthetic gene clusters across $B$.

40 apis strains suggest antifungal production via a Type I polyketide synthase. Secreted

41 metabolites from $B$. apis alone were sufficient to suppress fungal growth, supporting this

42 hypothesis. Together, these data suggest that $B$. apis protects bee brood from fungal

43 infection by the secretion of an antifungal metabolite. On the basis of this discovery, new

44 antifungal treatments could be developed to mitigate honey bee colony losses, and, in the

45 future, could address fungal epidemics in other species. 
Emerging fungal pathogens pose major threats to animal and plant populations ${ }^{2}$. Among insects, fungal pathogens are currently the most common causal agents of disease, and historically have plagued insect hosts for over 300 million years ${ }^{1,10}$. In recent years, fungal

49 pathogens have contributed to the unprecedented population decline of honey bees, causing

50 opportunistic infections in already stressed colonies ${ }^{3,4}$. Within the colony, the most susceptible

51 individuals are arguably the bee brood (larvae and pupae), which are exposed to fungal

52 pathogens, notably chalkbrood (Ascophaera apis) and stonebrood (Aspergillus flavus) ${ }^{11,12}$.

53 Although the spread of fungal disease among the brood can be limited by the hygienic behavior

54 of honey bee nurses ${ }^{13}$, this behavior does not prevent infection. However, brood fungal infections

55 in other insects are sometimes inhibited by the presence of bacterial symbionts ${ }^{14,15,8}$. Given that

56 honey bee brood are reared in the presence of a handful of bacterial taxa $a^{16,17}$, it is possible these

57 microbes play similar defensive roles. Indeed, worker honey bee pathogen susceptibility

58 correlates with changes in their microbiome composition and abundance ${ }^{18,19,20,21}$. Furthermore,

59 the presence of key microbiome members in worker bees can alter the prevalence of bacterial

60 diseases $22,23,24,25$. In aggregate, this evidence suggests that honey bee-associated bacteria can

61 defend against bacterial pathogens and may similarly protect the host from fungal disease.

63 Parasaccharibacter apium), an acetic-acid bacterium found in association with nectar and royal

64 jelly. Within the colony it is distributed across niches including larvae, the queen's gut, worker

65 hypopharyngeal glands, and nectar stores. Many of the niches it colonizes, particularly the

66 larvae, are susceptible to fungal infection and/or contamination, and its localization to these

67 niches may be indicative of a protective role. Furthermore, increased B. apis load is negatively

68 correlated with Nosema (a fungal pathogen) in honey bee adults, suggesting interactive effects. 
69 However, since B. apis is rarely found in adult guts, this interaction may be the result of B. apis-

70 fungal interactions in the diet and where brood are reared. Additionally, the mechanism by which

71 B. apis might interact with and/or suppress fungal pathogens is unknown.

Here we examined the potential of $B$. apis to prevent fungal infection in brood and the

73 bacterial genes underlying pathogen defense. To determine the impact of B. apis on fungal

74 colonization, we used two different insect pathogens in our assays: Beauveria bassiana, a

75 generalist pathogen that infects $70 \%$ of insect species, and A. flavus, an opportunistic pathogen

76 of honey bee brood. To determine the ability of B. apis to inhibit fungal growth in vitro, we

77 competed each fungal pathogen with one of five B. apis strains, isolated from apiaries in the US

78 (Fig 1a). In the presence of B. apis strains, fungal growth was either suppressed or completely

79 inhibited, (Fig 1b). To quantify fungal inhibition, we counted spores of B. bassiana or A. flavus

80 co-cultured with B. apis. The number of spores produced by both B. bassiana and A. flavus, was

81 reduced by an order of magnitude on average (Fig 1c), showing that B. apis can suppress growth

82 of both pathogens.

To test if $B$. apis is capable of preventing fungal infections in vivo, we collected larvae

84 from our apiary and reared them on a diet supplemented with either B. apis or a sterile media

85 control. Once reared to pupae, the cohort was inoculated with A. flavus or a sterile media control

86 and presence of infection was scored until adulthood (Fig 2a). Pupae that were supplemented

87 with $B$. apis as larvae were significantly more likely to resist fungal infection $\left(\chi^{2}=14.8, \mathrm{df}=1\right.$,

$88 \mathrm{p}<0.001$ ), with $66 \%$ of the cohort surviving to adulthood with no signs of infection (Fig $2 \mathrm{~b}, \mathrm{c}$ ).

89 In sharp contrast, without B. apis, no pupae survived to adulthood (Fig 2b, d). Interestingly, in

90 the $34 \%$ of $B$. apis-supplemented pupae that succumbed to fungal infection, the number of spores

91 produced was $68 \%$ on average $($ Fig $2 \mathrm{e} ; \mathrm{t}=2.9116, \mathrm{df}=8.4595, \mathrm{p}=0.02)$. Taken together, these 
92 results suggest that the presence of B. apis increases the host's likelihood of survival under

93 fungal challenge, while decreasing the pathogen's spore load and potential to spread infection to

94 new hosts.

95 To determine if $B$. apis produces antifungal metabolite(s), we incubated fungi in spent

96 media (SM) from B. apis, filtered to exclude bacterial cells and normalized for final optical

97 density reached (Fig 3a). Growth of both B. bassiana and A. flavus were significantly reduced by

98 spent media alone, indicating that B. apis-induced changes in the media are sufficient to suppress

99 fungal growth. To eliminate the possibility that fungal inhibition was mediated by acidification

100 of the media, A. flavus was cultured in media acidified to $\mathrm{pH}$ of 5.0 (the same $\mathrm{pH}$ of B. apis SM).

$101 \mathrm{pH}$ had no significant effect on fungal growth (Fig S3; $\mathrm{t}=-0.251, \mathrm{df}=35, \mathrm{p}=0.804)$. Therefore,

102 it is likely that B. apis inhibits fungi via secretion of an antifungal secondary metabolite(s). We

103 used antiSMASH ${ }^{26}$ to annotate secondary metabolite gene clusters in the genomes of all B. apis

104 strains used in this study and found that all strains have a conserved type 1 polyketide synthase

105 (T1PKS) region. Type 1 polyketide synthases are common among host-associated microbes and

106 produce macrolides which often have antifungal activity ${ }^{8,27,28,29}$. Additionally, all B. apis strains

107 contain an aryl polyene synthesis cluster. The commonly used antifungals amphotericin, nystatin

108 and pimaricin are all polyenes, suggesting that this gene cluster may also contribute to the

109 production of antifungal compound(s). Further functional characterization of these gene clusters

110 will help elucidate whether they play a role in the antifungal phenotype of $B$. apis. Considering

111 the antifungal activity of B. apis secreted metabolites in vitro and our genomic predictions, it is

112 likely that B. apis synthesizes and secretes a metabolite capable of inhibiting fungi.

113 Our results provide evidence that a honey bee-associated bacterium, B. apis, is capable of

114 suppressing two prevalent insect fungal pathogens both in vitro and in vivo, likely via the 
115 synthesis of an antifungal metabolite. Our in vitro results demonstrate antifungal activity in all

116 sampled strains of B. apis, with some variation between strains. Analysis of biosynthetic gene

117 clusters present across all strains of B. apis revealed two putative regions involved in antifungal

118 production: an aryl polyene synthetase and a T1PKS. Given that a significant proportion of

119 known bacterially-produced antifungals are polyketides ${ }^{8,27,28,29}$, the T1PKS is a promising

120 candidate region.

On the basis of our in vivo experiments, supplementing honey bee colonies with B. apis

122 may decrease colony losses due to fungal disease. Indeed, in the field, supplementation of $B$.

123 apis is correlated with a reduction in Nosema load in adult bees ${ }^{22}$. Beyond decreasing colony

124 losses and fungal load via direct inhibition of fungal infection, the presence of B. apis may limit

125 disease transmission by reducing the number of spores produced per infection. In addition, it

126 may suppress adult-specific pathogens, which could be transiently harbored in the larval diet

127 between adult hosts ${ }^{30}$.

Altering the prevalence of pathogenic fungi within managed honey bee colonies could

129 have further ecological consequences. Floral resources shared among diverse pollinators act as

130 transmission centers for fungi, both pathogenic and saprophytic ${ }^{31}$. Species-specific fungal

131 pathogens can be seeded in pollen and nectar sources ${ }^{32}$, after which diverse pollinators, including

132 native bees, can act as vectors to transmit the fungal pathogens to other floral sources, thereby

133 facilitating heterospecific transmission of fungal agents ${ }^{33}$. As a result of reduced spore loads

134 within colonies, the load of fungal pathogens deposited in local floral resources by foragers

135 might also decrease, and perhaps reduce heterospecific transmission and spillover events ${ }^{34}$. 
137 Competition assays were carried out with stationary cultures of B. apis normalized to the same

138 OD and $10^{3}$ spores of either fungal isolate in liquid or solid MRS media. The number of spores

139 produced was counted on a hemocytometer under a light microscope at 40x magnification.

140 Larvae were maintained on UV-sterilized larval diet and supplemented with stationary cultures

141 of B. apis. A total of $10^{3}$ spores of A. flavus were added to half the brood, five days into the

142 pupal phase. Presence of fungal infection was scored daily until adulthood. Spent media (SM) of

143 B. apis was obtained by spinning down stationary cultures and filtering out remaining bacterial

144 cells using a 0.25 um filter. $10^{3}$ spores of either fungal isolate were incubated in equal volumes

145 SM and fresh media; OD600 was used as proxy for fungal growth. Genomes for all strains were

146 downloaded from GenBank (see Table 1 for accession numbers) and re-annotated with

147 RAST ${ }^{35,36}$. The resulting GFF files and corresponding genome files were uploaded to

148 antiSMASH ${ }^{26}$ and results were compared across strains to determine conserved secondary

149 metabolite synthesis clusters.

151 1. St. Leger, R. J. \& Wang, C. Genetic engineering of fungal biocontrol agents to achieve 152 greater efficacy against insect pests. Applied Microbiology and Biotechnology 85, 901$153907(2010)$.

154 2. Fisher, M. C. et al. Emerging fungal threats to animal, plant and ecosystem health. (2012). doi:10.1038/nature10947

156 3. Brodschneider, R. et al. Multi-country loss rates of honey bee colonies during winter 157 2016/2017 from the COLOSS survey. J. Apic. Res. (2018).

158 doi:10.1080/00218839.2018.1460911 
4. Paxton, R. J. Does infection by Nosema ceranae cause 'Colony Collapse Disorder' in honey bees (Apis mellifera)? J. Apic. Res. 49, 80-84 (2010).

5. Fürst, M. A., McMahon, D. P., Osborne, J. L., Paxton, R. J. \& Brown, M. J. F. Disease associations between honeybees and bumblebees as a threat to wild pollinators. Nature 506, 364-366 (2014).

6. Roemer, T. \& Krysan, D. J. Antifungal drug development: challenges, unmet clinical needs, and new approaches. Cold Spring Harbor perspectives in medicine 4, (2014).

7. Williams, G. R., Shutler, D., Little, C. M., Burgher-Maclellan, K. L. \& Rogers, R. E. L. The microsporidian Nosema ceranae, the antibiotic Fumagilin-B ${ }^{\circledR}$, and western honey bee (Apis mellifera) colony strength. Apidologie 42, 15-22 (2011). rsc.li/chem-soc-rev Includes themed articles on chemical signaling at the eukaryotic/prokaryotic interface Defense contracts: molecular protection in insect-microbe symbioses. Chem. Soc. Rev 47, 1638

9. Kaltenpoth, M. Actinobacteria as mutualists: general healthcare for insects? parasitism by fungi supports a Cretaceous diversification of fungal-arthropod symbioses. Mol. Phylogenet. Evol. 49, 495-502 (2008).

11. Foley, K., Fazio, G., Jensen, A. B. \& Hughes, W. O. H. The distribution of Aspergillus spp. opportunistic parasites in hives and their pathogenicity to honey bees. Vet. Microbiol. 
169, 203-210 (2014).

12. Aronstein, K. A. \& Murray, K. D. Chalkbrood disease in honey bees. J. Invertebr. Pathol. 103, (2010).

13. Swanson, J. A. I. et al. Odorants that induce hygienic behavior in honeybees: Identification of volatile compounds in chalkbrood-infected honeybee larvae. J. Chem. Ecol. 35, 1108-1116 (2009).

14. Kaltenpoth, M., Göttler, W., Herzner, G. \& Strohm, E. Symbiotic bacteria protect wasp larvae from fungal infestation. Curr. Biol. 15, 475-479 (2005).

15. Flórez, L. V. et al. An antifungal polyketide associated with horizontally acquired genes supports symbiont-mediated defense in Lagria villosa beetles. Nat. Commun. 9, 2478 (2018).

16. Rokop, Z. P., Horton, M. A. \& Newton, I. L. G. Interactions between Cooccurring Lactic Acid Bacteria in Honey Bee Hives. Appl. Environ. Microbiol. 81, 7261-70 (2015).

17. Vojvodic, S., Rehan, S. M. \& Anderson, K. E. Microbial Gut Diversity of Africanized and European Honey Bee Larval Instars. PLoS One 8, 72106 (2013).

18. Erban, T. et al. Bacterial community associated with worker honeybees ( Apis mellifera) affected by European foulbrood . PeerJ(2017). doi:10.7717/peerj.3816

19. Erban, T. et al. Honeybee (Apis mellifera)-associated bacterial community affected by /631/158/855 /631/326/2565/855/38/23/38/22/38/47 article. Sci. Rep. (2017). 
20. Maes, P. W., Rodrigues, P. A. P., Oliver, R., Mott, B. M. \& Anderson, K. E. Diet-related gut bacterial dysbiosis correlates with impaired development, increased mortality and Nosema disease in the honeybee (Apis mellifera). Mol. Ecol. (2016). doi:10.1111/mec.13862

21. Raymann, K., Shaffer, Z. \& Moran, N. A. Antibiotic exposure perturbs the gut microbiota and elevates mortality in honeybees. PLoS Biol. (2017). doi:10.1371/journal.pbio.2001861

22. Corby-Harris, V. et al. Parasaccharibacter apium, gen. Nov., sp. Nov., Improves Honey Bee (Hymenoptera: Apidae) resistance to Nosema. J. Econ. Entomol. (2016). doi:10.1093/jee/tow012

23. Schwarz, R. S., Moran, N. A. \& Evans, J. D. Early gut colonizers shape parasite susceptibility and microbiota composition in honey bee workers. Proc. Natl. Acad. Sci.

24. Forsgren, E., Olofsson, T. C., Vásquez, A. \& Fries, I. Novel lactic acid bacteria inhibiting (2016). doi:10.1073/pnas.1606631113 native gut microbiota of honey bees. R. Soc. Open Sci. (2017). doi:10.1098/rsos.170003 pipeline. Nucleic Acids Res. 47, W81-W87 (2019). genomic contexts. Proc. Natl. Acad. Sci. U. S. A. 113, 12940-12945 (2016). 
wasp offspring. Nat. Chem. Biol. 6, 261-263 (2010).

223

224

225

226

227

228

229

230

231

232

233

234

235

236

237

238

239

240

29. Chevrette, M. G. et al. The antimicrobial potential of Streptomyces from insect microbiomes. Nat. Commun. 10, 516 (2019).

30. Folly, A. J., Koch, H., Stevenson, P. C. \& Brown, M. J. F. Larvae act as a transient transmission hub for the prevalent bumblebee parasite Crithidia bombi. J. Invertebr. Pathol. 148, 81-85 (2017).

31. Graystock, P., Goulson, D. \& Hughes, W. O. H. Parasites in bloom: Flowers aid dispersal and transmission of pollinator parasites within and between bee species. Proc. R. Soc. B Biol. Sci. 282, (2015).

32. Graystock, P. et al. The Trojan hives: Pollinator pathogens, imported and distributed in bumblebee colonies. J. Appl. Ecol. (2013). doi:10.1111/1365-2664.12134

33. Hedtke, S. M., Blitzer, E. J., Montgomery, G. A. \& Danforth, B. N. Introduction of nonnative pollinators can lead to trans-continental movement of bee- Associated fungi. PLoS One 10, (2015).

34. Plischuk, S. et al. South American native bumblebees (Hymenoptera: Apidae) infected by Nosema ceranae (Microsporidia), an emerging pathogen of honeybees (Apis mellifera). Environ. Microbiol. Rep. 1, 131-135 (2009).

35. Aziz, R. K. et al. The RAST Server: Rapid annotations using subsystems technology. BMC Genomics 9, (2008).

36. Overbeek, R. et al. The SEED and the Rapid Annotation of microbial genomes using Subsystems Technology (RAST). Nucleic Acids Res. 42, D206-D214 (2014) 
a.
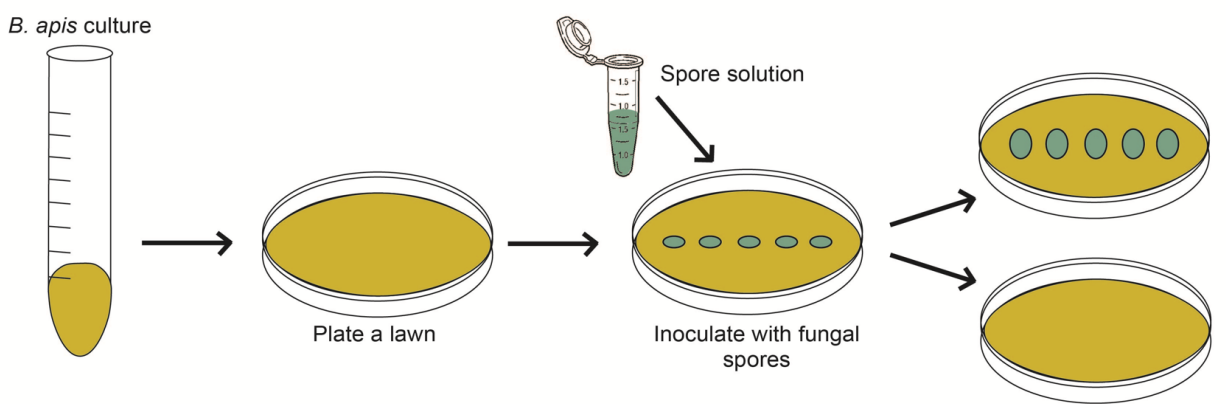

Score for presence/absence of growth

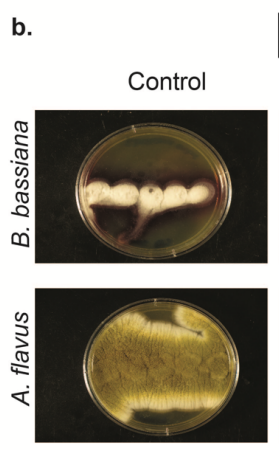

c.
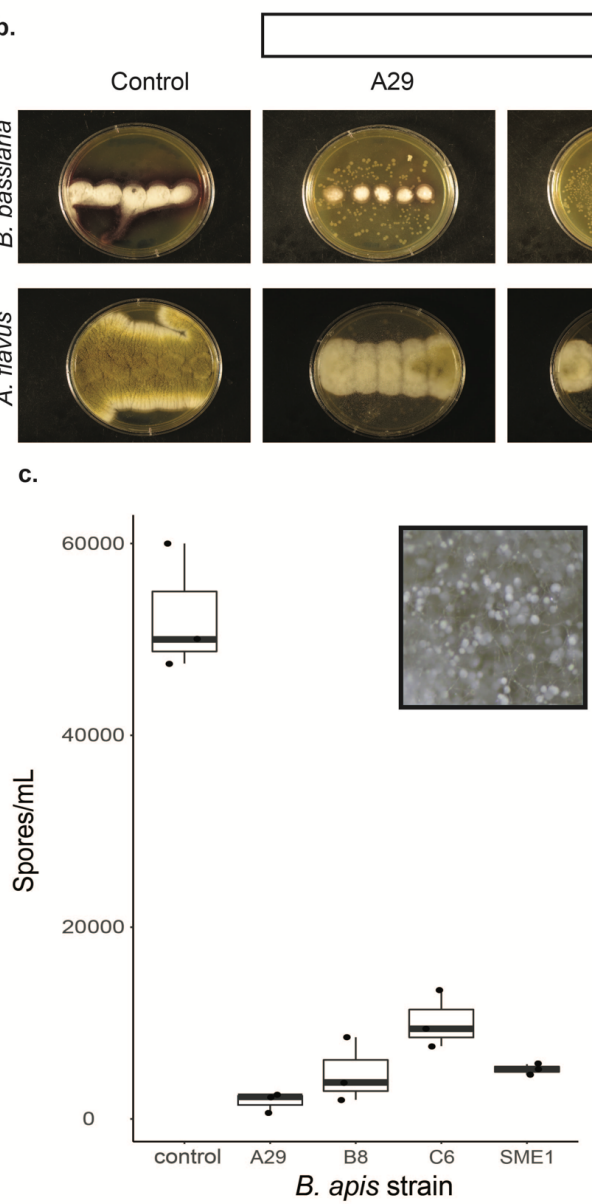

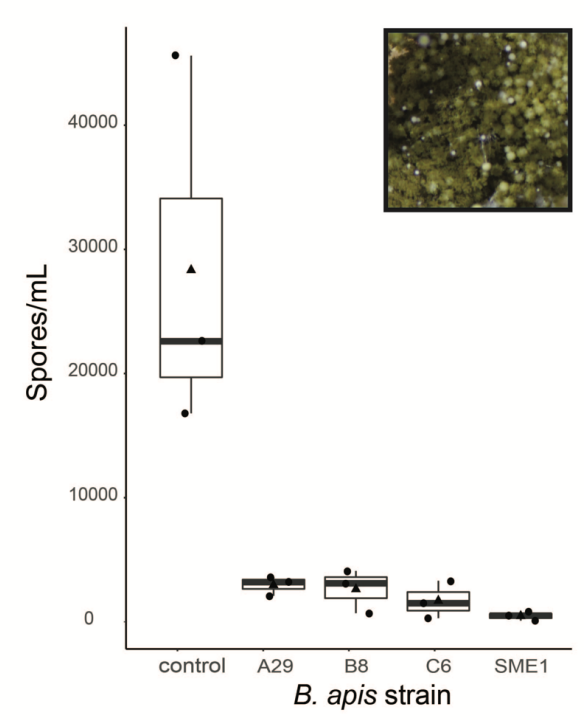

d.

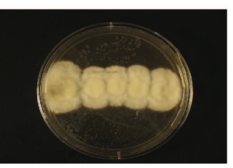

Figure 1: B. apis outcompetes fungal pathogens in vitro. a, The ability of each fungal isolate to grow on a $B$. apis lawns was qualitatively assayed. $\mathbf{b}$, Compared to fungal controls, the presence of $B$. apis either suppressed or completely inhibited fungal growth, depending on strain identity. $\mathbf{c}$, When co-cultured in liquid media, the presence of $B$. apis strongly reduced the number of spores produced by B. bassiana (A29: $\mathrm{t}=13.114$, $\mathrm{df}=2, \mathrm{p}=0.19 ; \mathrm{B} 8: \mathrm{t}=11.147$, $\mathrm{df}$ $=3, \mathrm{p}=0.006 ; \mathrm{C} 6: \mathrm{t}=10.121, \mathrm{df}=2.7, \mathrm{p}=0.011 ; \mathrm{SME} 1 \mathrm{t}=12.352, \mathrm{df}=2, \mathrm{p}=0.025)$ and $A$. flavus (A29: $\mathrm{t}=2.8807, \mathrm{df}=2, \mathrm{p}=0.40 ; \mathrm{B} 8: \mathrm{t}=2.9033, \mathrm{df}=2, \mathrm{p}=0.39 ; \mathrm{C} 6: \mathrm{t}=3.0137, \mathrm{df}=2$, $\mathrm{p}=0.37$; SME1: $\mathrm{t}=3.1679, \mathrm{df}=2, \mathrm{p}=0.34$ ), depending on $B$. apis strain identity. 
a.

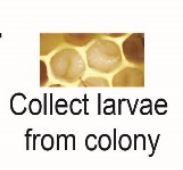

B. apis + B. apis -

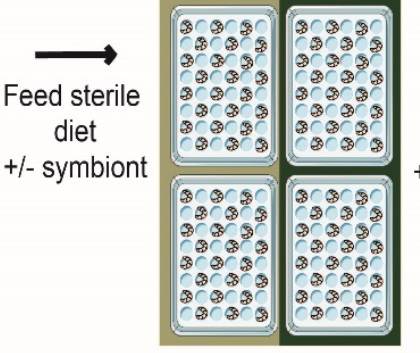

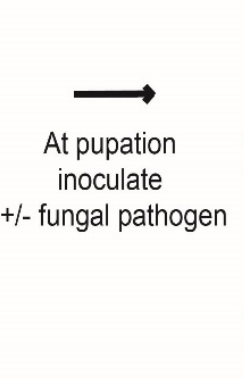
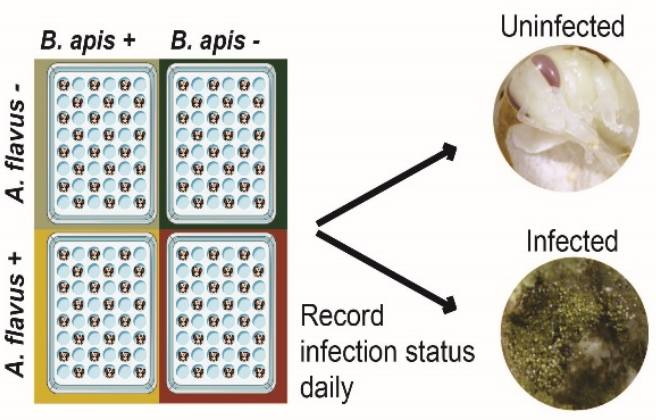

b.

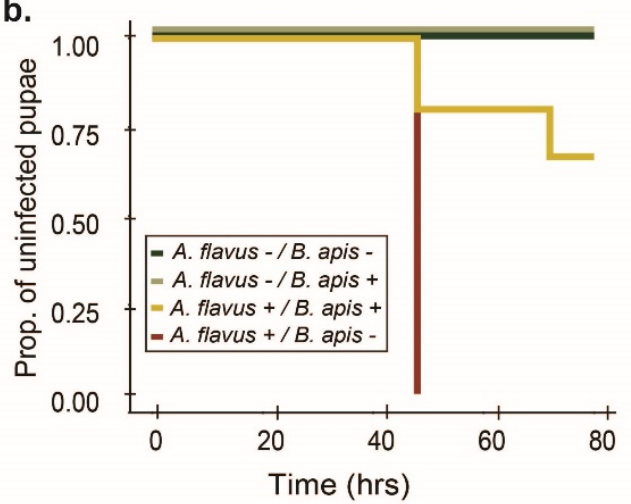

c.

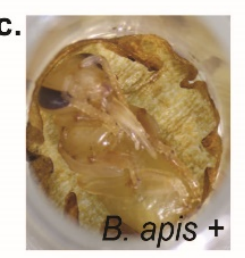

d.

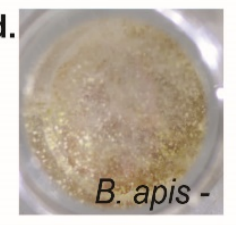

e.

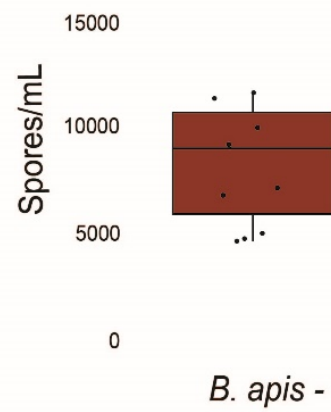

B. apis +

Figure 2: Bee brood supplemented with $B$. apis are less susceptible to infection with

A. flavus. a, First instar larvae $(\mathrm{n}=45)$ collected from the apiary were reared on sterile larval diet $+/-B$. apis (AJP2). Five days after pupation, each pupa was inoculated with $10^{3}$ spores of A. flavus $+/-$ B. apis or $0.01 \%$ Triton X-100 as a control. $\mathbf{b}$, Of the pupae inoculated with A. flavus, those without $B$. apis all showed signs of infection by $48 \mathrm{hrs} \mathbf{d}$, whereas $66 \%$ of those with $B$. apis never developed infections $\left(\chi^{2}=14.8, \mathrm{df}=1, \mathrm{p}<0.001\right) \mathbf{c}$. e, Pupae with B. apis that did become infected had lower intensity infections, producing significantly $(\mathrm{t}=5.5052, \mathrm{df}=$ $5.5751, \mathrm{p}=0.002$ ) fewer spores than those without $B$. apis. 
a.

B. apis culture

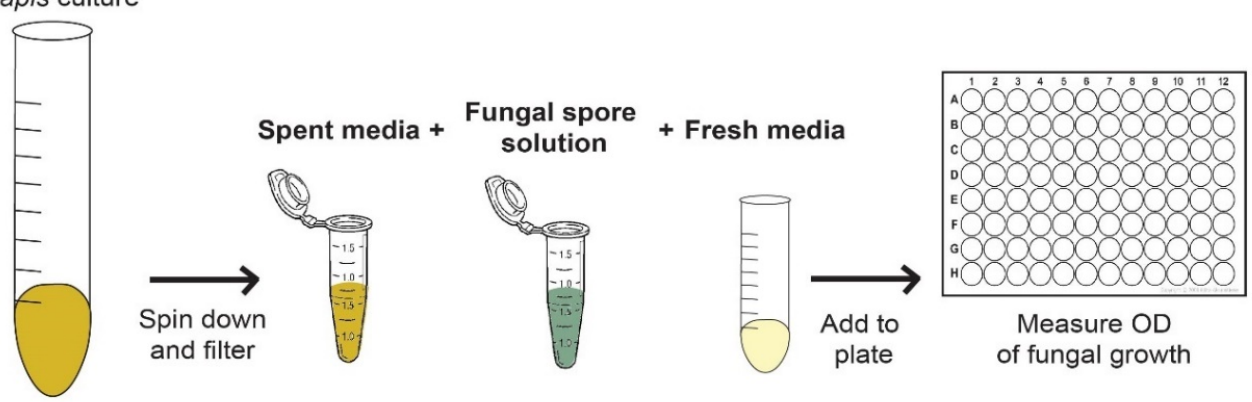

b.
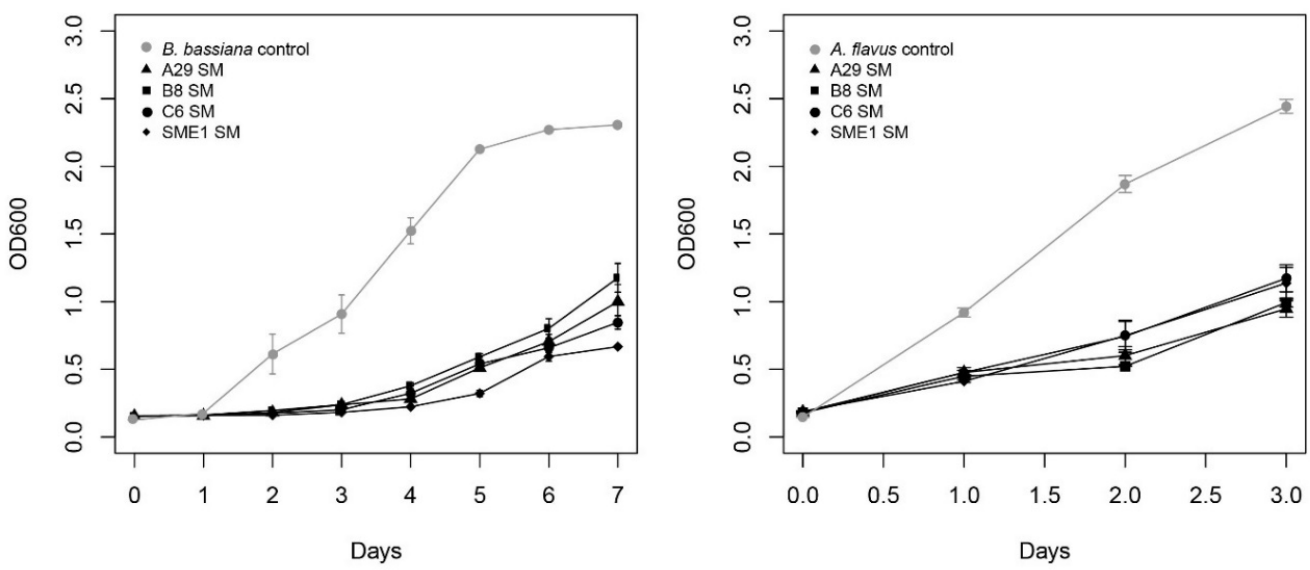

c.

Type 1 polyketide synthase

$10 \overline{00} \mathrm{bp}$

Arylpolyene

Figure 3: Fungal inhibition is mediated by $B$. apis secreted metabolites. a, Spores of fungal isolates were incubated in spent media (SM) from B. apis cultures. b, The growth of both B. bassiana (A29: $\mathrm{t}=-15.315, \mathrm{df}=119, \mathrm{p}<0.001 ; \mathrm{B} 8: \mathrm{t}=-13.925, \mathrm{df}=119, \mathrm{p}<0.001 ; \mathrm{C} 6: \mathrm{t}=$ $-13.202, \mathrm{df}=119, \mathrm{p}<0.001 ;$ SME1: $\mathrm{t}=-11.963, \mathrm{df}=119, \mathrm{p}<0.001)$ and A. flavus $(\mathrm{A} 29: \mathrm{t}=-11$ $.398, \mathrm{df}=59, \mathrm{p}<0.001 ; \mathrm{B} 8: \mathrm{t}=-13.022, \mathrm{df}=59, \mathrm{p}<0.001 ; \mathrm{C} 6: \mathrm{t}=-13.282, \mathrm{df}=59, \mathrm{p}<0.001$; SME1: $\mathrm{t}=-11.261, \mathrm{df}=59, \mathrm{p}<0.001)$ in SM was strongly reduced compared to the control, suggesting secreted metabolites from B. apis mediate fungal inhibition. c, Genomic architecture of the type 1 polyketide synthase and arylpolyene secondary metabolite gene clusters identified by antiSMASH; gene models are colored based on putative function within the cluster and are oriented to show direction of transcription 


\section{Methods}

\section{Isolates and culturing}

All bacterial strains of $B$. apis and were obtained by sampling either nectar or larvae_(Table

1). Isolates were acquired from our apiary or from Leibniz-Institut DSMZ. All cultures were maintained at $25^{\circ} \mathrm{C}$ with $80 \% \mathrm{RH}$ or $34^{\circ} \mathrm{C}$ with ambient humidity respectively on PDA or $\mathrm{X}-100$, agitating with a cell scraper, and suspending the spores in the solution.

Table 1: Sampling of B. apis strains

\begin{tabular}{|l|l|l|l|l|}
\hline species & strain & origin & sample & number \\
\hline B. apis & AJP2 & NC & nectar & N/A \\
\hline B. apis & SME1 & IN & nectar & GCA_009362775.1 \\
\hline B. apis & A29 & AZ & larvae & GCA_002917995.1 \\
\hline B. apis & B8 & AZ & larvae & GCA_002917945.1 \\
\hline B. apis & C6 & AZ & larvae & GCA_002917985.1 \\
\hline
\end{tabular}

294 B. apis strains were grown to their maximal OD, and all strains were normalized to the 295 lowest OD value by diluting in fresh media. A lawn of B. apis was created by plating $100 \mu \mathrm{L}$ 296 of normalized culture on MRS agar plates. The plate was then inoculated with $10^{3}$ spores of 
course of three to seven days (depending on isolate) the presence of hyphal/conidia growth

299 was monitored.

$300 \quad$ Competition assays

301 B. apis strains were grown to their maximal OD, and all strains were normalized to the

B02 lowest OD value by diluting in fresh media. $10^{3}$ spores of each fungal isolate were incubated 303 in $100 \mu 1$ of density-normalized B. apis culture or $100 \mu 1$ of fresh media. Fungal growth was

304 monitored daily and once controls showed sporulation, spore counts were quantified for each

$305 \quad$ well via hemocytometer.

$306 \quad$ Larval collection and in vivo infections

307 Late first instars were grafted from our apiary at Indiana University Research and Teaching

308 Preserve into queen cups filled with UV-sterilized worker diet prepared as outlined in

309 Schmel et. al, $2016^{37}$. B. apis supplemented groups were given diet with a ratio of 1:4

310 stationary $(\mathrm{OD}=1.0)$ B. apis in MRS to worker diet. This bacterial load was between $2 \mathrm{x}$

B11 $10^{6}$ and $6 \times 10^{6}$ cells $/ \mathrm{mL}$. Control groups were given diet with a ratio of 1:4 axenic MRS

312 media to worker diet. After 5 days in larval diet, pre-pupae were transferred to new wells

313 after either MRS or B. apis in MRS was added. Five days into pupal development,

B14 individuals were inoculated with $10^{3}$ spores of $A$. flavus in $0.01 \%$ Triton X-100 or an equal

315 volume of $0.01 \%$ Trition X-100 as a control. B. apis-supplemented groups were co-

B16 inoculated with one final dose of the bacterium $\left(10^{4}\right.$ cells $)$; controls received the same

317 volume of MRS. Presence of infections (as evidenced by hyphae penetrating through the

318 cuticle and/or spore production) was scored daily until adulthood.

319 Analysis of biosynthetic gene clusters (BGCs) 
320 Genomes for all strains were downloaded from GenBank (see Table 1 for accession

321 numbers) and re-annotated with RAST ${ }^{3536}$. The resulting GFF files and corresponding

322 genome files were uploaded to antiSMASH ${ }^{26}$ and results were compared across strains to

323 determine conserved secondary metabolite synthesis clusters. Gene model figures were

324 visualized and adapted for publication using $\mathrm{R}^{38}$.

$325 \quad$ In vitro antifungal assay

326 To obtain spent media, strains were grown to their maximal OD (0.6-0.25), and all strains

327 were normalized to the lowest OD value by diluting in fresh media. Cultures were spun down

328 at $9,000 \mathrm{rpm}$ for $5 \mathrm{~min}$ and the supernatant filtered through a $0.2 \mu \mathrm{m}$ filter to remove

329 bacterial cells. Spent media and fresh media were added to a multi-well plate in equal

B30 volumes and $10^{3}$ spores from spore stock solutions were added. Growth was measured daily

331 by assaying $O D_{600}$. A positive control included spores in fresh media alone used to compare

332 to treatment groups with spent media. Optical densities of spent media alone were monitored

333 to ensure no bacterial growth occurred. Assay plates were incubated at the appropriate

334 temperature for the fungal isolate used. Since B. apis acidifies the media from a $\mathrm{pH}$ of 5.5 to

$335 \quad 5.0$, controls of MRS media reduced to $\mathrm{pH} 5.0$ with $\mathrm{HCl}$ were included.

$336 \quad \underline{\text { Statistical analyses }}$

337 All statistical analyses were performed in $\mathrm{R}^{38}$. Spore counts of fungal isolates in the presence

338 of B. apis were compared to controls with unequal variance, two sample $\mathrm{t}$ tests; $\mathrm{p}$-values

339 were Bonferroni-corrected for multiple comparisons across strains. In vivo infections are

340 displayed as Kaplan-Meier survival curves. B. apis + /- infected treatments were compared

341 with a long-rank test using R package, "survminer" 39 . Interactive effects of $B$. apis SM on 
growth of fungi over time were determined with a generalized linear model of OD, time, and

343 strain identity.

Data and code availability: All genomic data used in this manuscript are publicly available

345 through NCBI and listed in Table 1.

\section{$346 \quad$ Methods References}

347 37. Schmehl, D. R., Tomé, H. V. V, Mortensen, A. N., Martins, G. F. \& Ellis, J. D. Protocol 348 for the in vitro rearing of honey bee (Apis mellifera L.) workers. J. Apic. Res. 55, 113$349 \quad 129(2016)$.

350 38. R Core Team. R: A Language and Environment for Statistical Computing. (2018).

351 39. Biecek, A. K. and M. K. and P. survminer: Drawing Survival Curves using 'ggplot2'. R package version 0.4.6 (2019).

353 40. Pruesse, E., Peplies, J. \& Glöckner, F. O. SINA: Accurate high-throughput multiple sequence alignment of ribosomal RNA genes. Bioinformatics 28, 1823-1829 (2012).

355 41. Stamatakis, A. RAxML version 8: a tool for phylogenetic analysis and post-analysis of large phylogenies. Bioinformatics 30, 1312-1313 (2014).

42. FigTree. Available at: http://tree.bio.ed.ac.uk/software/figtree/. (Accessed: 6th December 2019)

43. Li, L., Stoeckert, C. J. \& Roos, D. S. OrthoMCL: Identification of ortholog groups for eukaryotic genomes. Genome Res. 13, 2178-2189 (2003).

44. Katoh, K. MAFFT: a novel method for rapid multiple sequence alignment based on fast Fourier transform. Nucleic Acids Res. 30, 3059-3066 (2002). 
Acknowledgements: This work was funded by a Project Apis $m$. grant to ILGN and a USDA

$364 \quad$ NIFA to EAS.

365 Author contributions: Conception and design of the work, ILGN and DLM, acquisition,

366 analysis, or interpretation of data, EAS and DLM, drafted and revised the manuscript, DLM,

367 EAS, ILGN.

$368 \quad$ Competing interests:

$369 \quad$ ILGN and DLM are co-founders of VitaliBee, a company based partly on the discovery

370 described herein.

$371 \quad$ Additional information

372 Supplementary information is available for this paper at:

373 Correspondence and requests for materials should be addressed ILGN. 
bioRxiv preprint doi: https://doi.org/10.1101/2020.0121.914325; this version posted January 23, 2020. The copyright holder for this preprint (which was not certified by peer review) is the author/funder, who has granted bioRxiv a license to display the preprint in perpetuity. It is made available under aCC-BY-NC-ND 4.0 International license.

\section{Supplemental Data}

396

a.

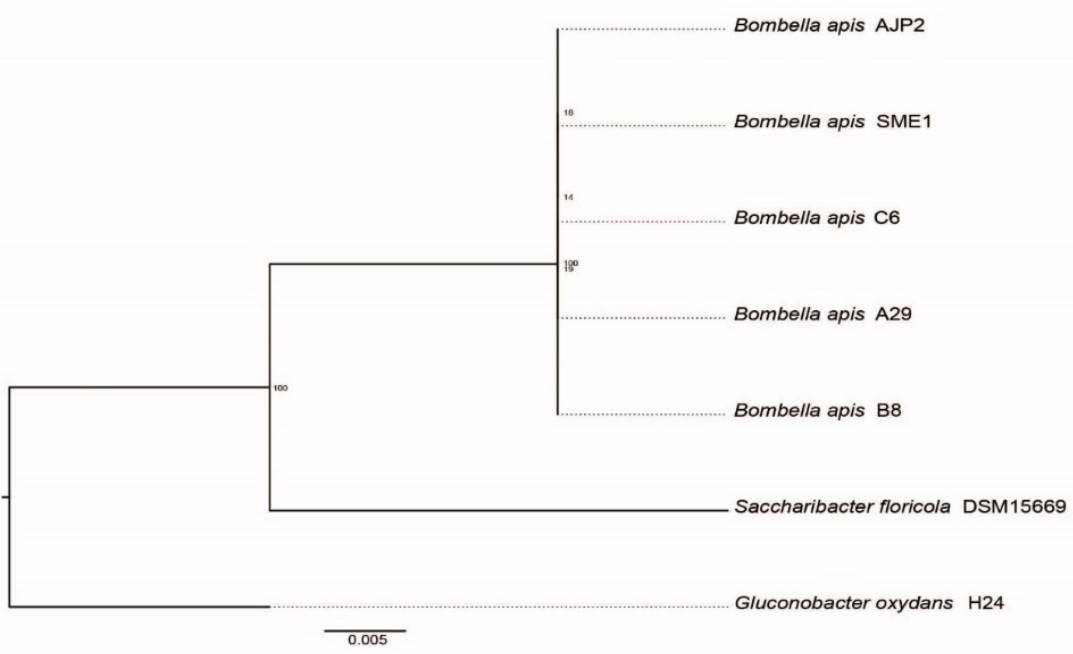

b.

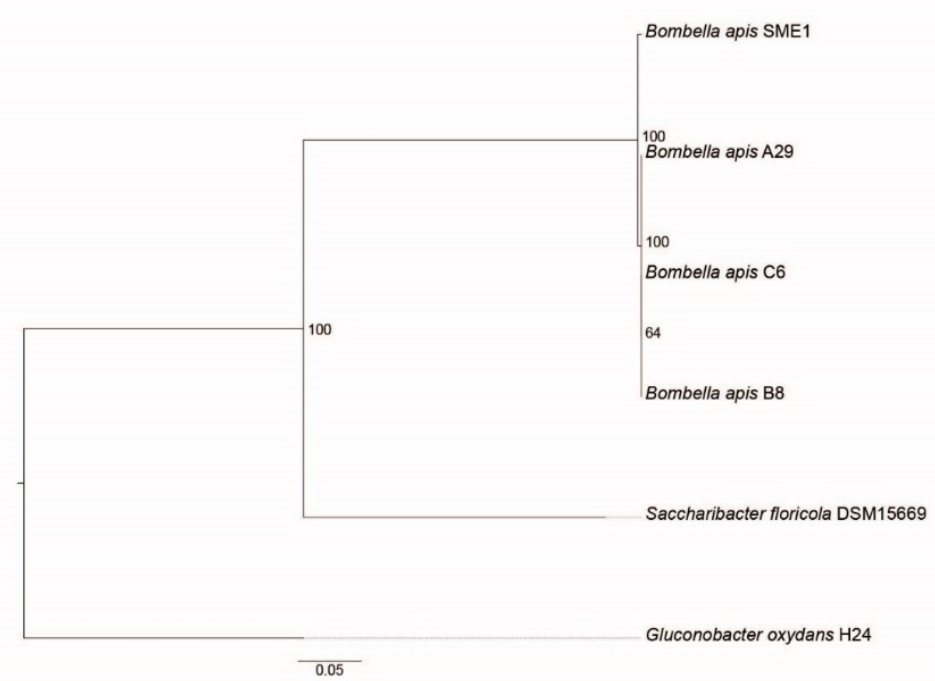


Supplementary Figure 1: A. Maximum-likelihood 16S rRNA gene sequence tree for strains used in this study. Saccharibacter floricola and Gluconobacter oxydans were used as outgroups. Sequences were downloaded from GenBank and aligned with the SINA aligner ${ }^{40}$. The tree was constructed with RAxML ${ }^{41}$ and visualized with FigTree ${ }^{42}$. Numbers at nodes represent bootstrap support from 1000 bootstrap pseudoreplicates. B. Core-ortholog maximum-likelihood phylogeny. All genomes were downloaded from GenBank and core orthologs were identified using OrthoMCL ${ }^{43}$. Alignments of core orthologs were made using MAFFT ${ }^{44}$ and concatenated together. As above, the tree was constructed with RAxML ${ }^{41}$ and visualized with FigTree ${ }_{-}^{42}$. Numbers at nodes represent bootstrap support from 1000 bootstrap pseudoreplicates.

a.

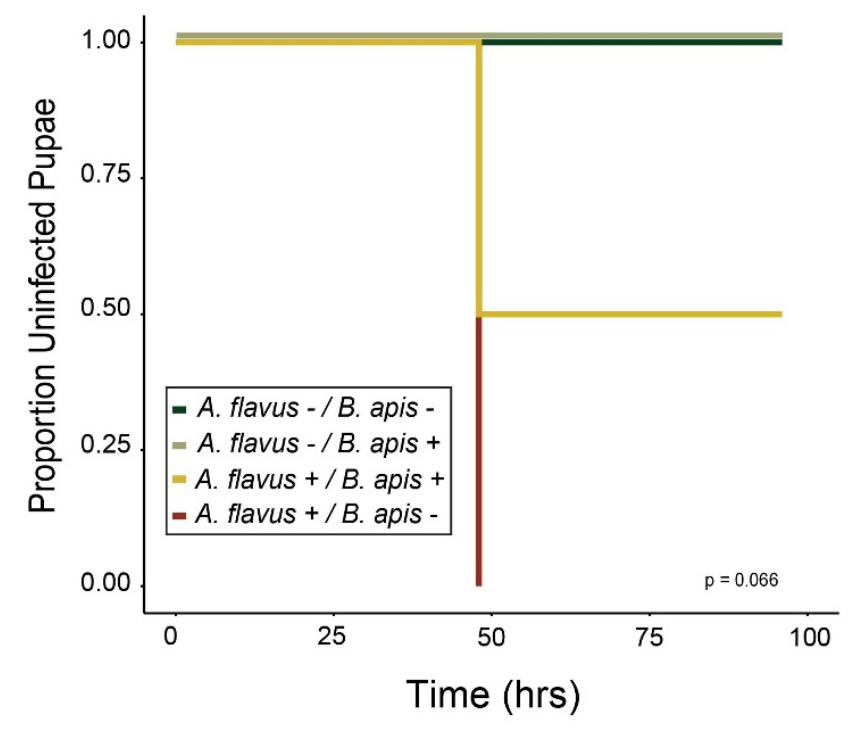

b.

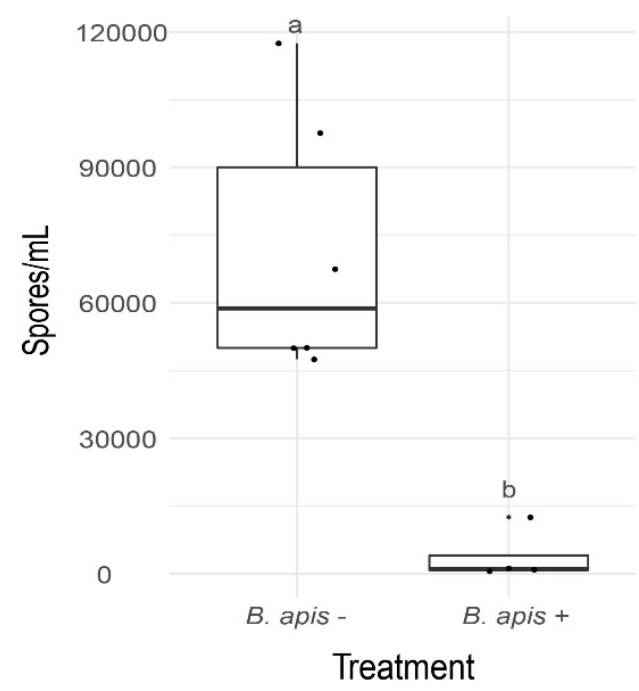
B. apis strain identity. a, First instar larvae $(\mathrm{n}=20)$ collected from the apiary were reared on sterile larval diet $+/-B$. apis (A29). Five days after pupation, each pupa was inoculated with $10^{3}$ spores of $A$. flavus $+/-$ B. apis or $0.01 \%$ Triton X-100 as a control. Pupae supplemented with A29 were more likely to survive to adulthood $\left(\chi^{2}=3.4, \mathrm{df}=1, \mathrm{p}=0.07\right) \mathbf{b}$, Presence of $B$. apis (A29) significantly reduced $(\mathrm{t}=5.5052, \mathrm{df}=5.5751, \mathrm{p}=0.001914)$ sporulation in infected pupae 


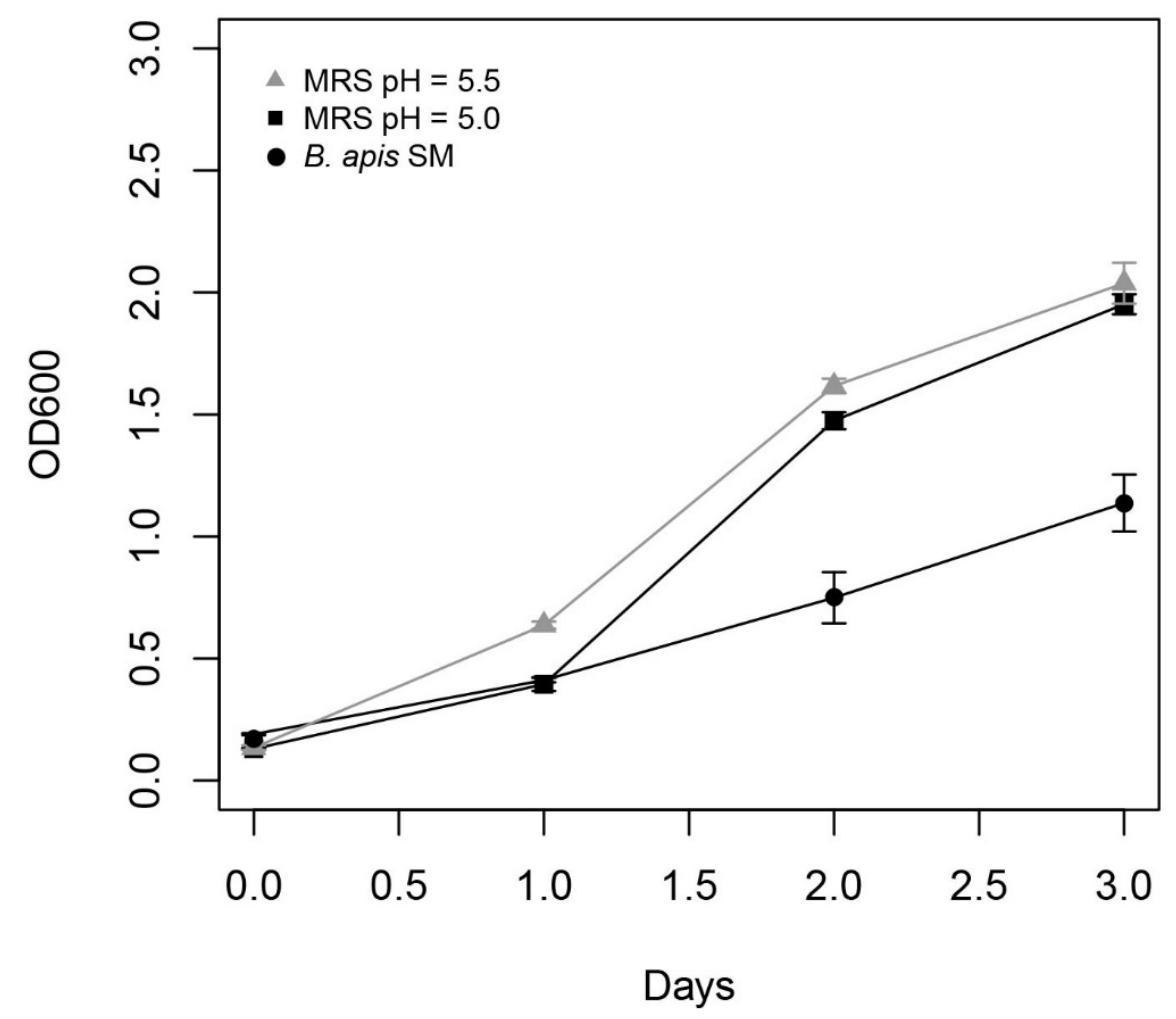

425 Supplemental Figure 3: Fungal inhibition by $\mathbf{S M}$ is not pH-mediated. B. apis (A29) reduces MRS media from a $\mathrm{pH}$ of 5.5 to 5.0. Spent media from B. apis at $\mathrm{pH} 5.0$ significantly reduced fungal growth $(\mathrm{t}=-6.111, \mathrm{df}=35, \mathrm{p}<0.001)$ while MRS media reduced to a $\mathrm{pH}$ of 5.0 using $\mathrm{HCl}$ did not significantly reduce growth $(\mathrm{t}=-0.251, \mathrm{df}=35, \mathrm{p}=0.804)$. 